\section{Evidence for a two-process theory of problem solving*}

\author{
JOHN BENJAFIELD \\ Brock University, St. Catharines, Ont., Canada
}

A two-process theory of problem solving, which holds that $\mathbf{S}$ derives a solution to a problem either from his representation of the goal (G) or from his representation of the problem materials (PM), was presented. The ease with which $\mathrm{S}$ could solve the problem through an inspection of PM and the number of steps necessary to deduce a subgoal from $G$, as given in the instructions, were both varied in a 2 by 2 design. The former variable had an effect only when $G$ was vague and the latter, an effect only with difficult PM. The results were taken as supporting a two-process theory.

When $S$ attempts to solve a problem, his effort is directed toward transforming an initial situation-the problem materials ( $P M$-into a final situation - the goal (G). As Berlyne (1965, ppp. 101-107) notes, one of the classical problems in the psychology of thinking is how these two aspects of the problem situation act to determine the problem solver's behavior. The purpose of the present study is to examine experimentally a theory of the interaction of G and PM.

Theories of this interaction fall into two classes: single-process theories (e.g., Davis, 1966) and two-process theories (e.g., Maier, 1945). The essence of the former theory is that "from the problem stimuli presented, and the instructions concerning the goal, $\mathrm{S}$ generates his own hierarchy of response alternatives which he sequentially tests and rejects until the correct response, or correct combination of responses, is rewarded via a solving of the problem [Davis, 1966 , p. 41].'

An alternative theory is that $G$ and PM need not operate conjointly but can function in essentially disjunctive ways. A model of this disjunctive action is given in Fig. 1. The PM are represented by $\mathrm{S}$ as having a hierarchy of functions, or uses $(\mathrm{f} 1-\mathrm{f} 4)$. The correct function (f4) is not the most obvious one (otherwise, a problem would not exist for $S$ ). Through a consideration of the goal (G), $S$ can derive a subgoal (SG), through whose solution the problem can also be solved. [For a discussion of the process of subgoal utilization, see Duncker (1945, p. 23) and de Groot (1965, p. 48).] Consideration of SG leads $S$ to perform $f 5$, a new function not given through an inspection of PM. Both $\mathrm{f} 4$ and $\mathrm{f} 5$ constitute solutions, and, for problems which have but a

*The author is grateful to Dr. M. Simmel, Brandeis University, for many helpful suggestions during the course of this study.

single solution, f4 and f5 will be behaviorally identical. However, they will be phenomenally different. From the standpoint of $E$, the criterion of this difference will be the reason $S$ gives for performing $\mathrm{f} 4$ or $\mathrm{f} 5$. Ss performing $\mathrm{f} 4$ should give as their reason for doing so simply that they saw $\mathrm{f} 4$ as possible to perform with $\mathrm{PM}$. However, Ss performing f5 should report that they performed $f 5$ in order to reach $\mathrm{SG}$.

In general, the probability of $S$ solving the problem can be increased in two ways. On the one hand, moving $\mathrm{f} 4 \mathrm{up}$ in the hierarchy of functions (making f4 more obvious) should make the problem easier. On the other hand, increasing the likelihood of S's discovering SG should also increase the likelihood of S's performing $f 5$.

Reid (1951) has shown that the likelihood that $\mathbf{S}$ will deduce $\mathrm{SG}$ from $G$, as given in the instructions, decreases with the number of inferential steps necessary to deduce SG. Within a given problem-solving situation, one can distinguish between specific goals (Sp) and nonspecific goals (NSp), the former requiring relatively few steps to reach a subgoal, the latter requiring relatively many. Ss given $S p$ should be more likely to perform f5 than Ss given NSp. Conversely, Ss given NSp should be more likely to solve the problem by finding f4, i.e., through "blind variation" (Campbell, 1960) of their responses to $P M$ until they find a solution. Thus, NSp Ss should make more errors than $\mathrm{Sp} S \mathrm{Ss}$ prior to a solution. Making $\mathrm{f} 4$ more obvious should increase the likelihood of NSp Ss' solving the problem, but, since Sp Ss are more likely to deduce SG and perform $\mathrm{f} 5$, making $\mathrm{f} 4$ more obvious should make less of a difference to them.

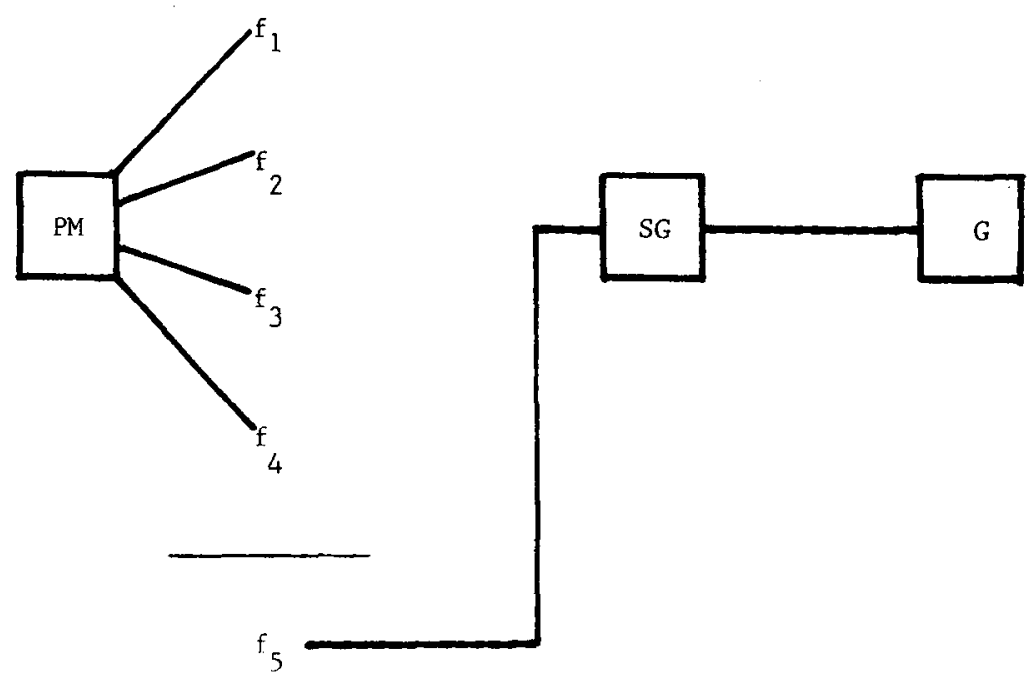

Fig. 1. Relation of goal and problem material representations. 
Table 1

Proportion of $S s$ in Each Group Solving the Problem

\begin{tabular}{lcccr} 
Group & NSp-T & NSp-E & Sp-T & Sp-E \\
\hline $\begin{array}{l}\text { Propor- } \\
\text { tion } \\
\text { Solving }\end{array}$ & .25 & .83 & 1.00 & .58 \\
$N$ & 12 & 12 & 12 & 12 \\
\hline
\end{tabular}

(3) move that half through an arc such that the squares meet face to face. It is in the second phase of this transformation that a difficulty arises which hinders the solution of the problem. S correctly sees the table surface as impenetrable but falsely concludes that the apex of the triangle must penetrate the table surface if the squares are to be placed face to face. Since the apex of the triangle cannot penetrate the table surface, $S$ abandons this transformation in midstream. Consequently, he fails to solve the problem.

This difficulty was eliminated by elevating each half of the tetrahedron $1 \mathrm{ft}$ off the table by means of a transparent plastic tube. The presentation of the materials, then, was either elevated (E) or on the table (T). Each half was always presented with one trapezoid parallel to the table top and the square facing $S$. The $E$ condition makes the solution more obvious by allowing $S$ to see it as possible. Conversely, the $T$ condition makes the correct transformation less obvious.

Goal specificity was manipulated by either telling $S$ to make an object which had four surfaces or to make a pyramid which had four surfaces. The first instruction requires two inferences to arrive at the subgoal, "make triangles." First, $S$ must infer that all four-surfaced objects are four-surfaced pyramids; second, he must infer that all four-surfaced pyramids have only triangular surfaces. If $S$ is told to make a four-surfaced pyramid, he need only make one inference to reach the appropriate subgoal. These two instructions constituted the nonspecific (NSp) and the specific ( $\mathrm{Sp}$ ) goals, respectively.

\section{PROCEDURE}

The two variables (goal specificity and presentation of materials) were cast in a 2 by 2 design. Six men and six women served as Ss in each of the four conditions. The Ss were instructed as follows: "Here is a problem I would like you to solve. It goes as follows. Here are two identical objects. Each object has two identical triangles, two identical trapezoids, and a square. I want you to think of a way of putting these objects together so as to make (1) an object with four surfaces, (2) a pyramid with four surfaces [ $\mathrm{S}$ is given either (1) or (2)] You are not allowed to touch the objects. While you go about solving the problem, I would like you to think aloud. It is very important that you think aloud as much as possible, so that I get as complete an account of your thinking as I can."

Everything $S$ said in the course of problem solving was written down by E. $S$ was given $45 \mathrm{~min}$ to solve the problem, although, if he asked, he was told he had as much time as he liked. No S was allowed to "give up" until he had worked on the problem for $20 \mathrm{~min}$.

\section{RESULTS \\ Proficiency}

The proportion of Ss solving the problem in each group is given in Table 1. The statistical significance of the obtained differences was tested for the distribution as a whole $\left(U_{o}^{\prime}=21.36, d f=3, p<.001\right)$ and for all pairs, using Cohen's (1967) technique. Multiple comparisons between the proportions showed that a significantly greater proportion of Ss in Group Sp-T solved the problem than in Group NSp-T and that a significantly greater proportion of Ss in Group NSp-E solved the problem than in Group NSp-T.

\section{Errors}

An error was scored whenever $\mathbf{S}$ described an erroneous conjunction of the two halves of the tetrahedron, provided the description was not prefaced by the phrase, "I've already tried ...." and was thus an obvious recall of a previous error. The vast majority of errors were "identity errors," consisting of face-to-face placement of two identical surfaces (i.e., trapezoid to trapezoid, triangle to triangle, square to square). The median number of identity errors of the $44 \mathrm{Ss}$ who completed the problem (i.e., did not "give up") was eight. The proportion of Ss below this median in each experimental group is given in Table $2 \quad\left(U_{0}^{\prime}=21.62, \quad \mathrm{~d} f=3\right.$, $\mathbf{p}<.001$ ). Multiple comparisons showed that the proportion of Ss below this median is greater in Sp-T than in either Group NSp-T or Group NSp-E.

\section{Type of Solution}

A deductive solution was defined as occurring when $S$ verbalized the necessity of making triangles and then described placing a triangle adjacent to a trapezoid as the solution. Deductive solutions occur almost twice as frequently as any other solution. Table 3 shows the obtained differences in the distribution of these solutions $\left(\mathrm{U}_{\mathrm{o}}^{\prime}=24.36, \quad \mathrm{df}=3, \quad \mathrm{p}<.001\right.$ ) Multiple comparisons showed that the proportion of deductive solutions made by Group $\mathrm{Sp}-\mathrm{T}$ was greater than that of either Group NSp-T or NSP-E. DISCUSSION

The fact that both Groups NSp-E and NSp-T made significantly more identity errors than Group Sp-T, while both Groups Sp-T and NSp-E contained a greater proportion of $\mathrm{Ss}$ who solved the problem than did Group NSp-T is consistent with the hypothesis that two types of solution were operative: those determined by the problem materials, and those determined by an analysis of the goal. Ss in the NSp groups attempted to solve the problem by using the functional representation of the problem materials and, as a result, made many errors. This procedure worked well for NSp-E Ss but not for NSp-T Ss. Conversely, Sp-T Ss apparently did not utilize the functional representation of the problem materials as much and, consequently, made fewer errors. Their solutions were more determined by a goal analysis.

Further confirmation of these points comes from the protocol analysis. In general, specific goal groups showed a preponderance of deductive solutions. Altogether, 14 deductive solutions were produced by the two groups given the specific goal, and only one single deductive solution came from the $S s$ who received the nonspecific goal.

At first glance, the results of Group Sp-E seem somewhat anomalous. This group failed to do better than either NSp group in proficiency. The present study proposed that the goal and problem materials do not act as a unit. If they
Table 2

Proportion of Ss in Each Group Below Median Identity Error Score

\begin{tabular}{llllll}
\hline Group & NSp-T & NSp-E & Sp-T & Sp-E
\end{tabular}

Proportion

Below

.10

.36

.92

.55

Median

10

11
12

11
Table 3

Proportion of $\mathrm{Ss}$ in Each Group Solving Deductively

\begin{tabular}{lcccr}
\multicolumn{1}{c}{ Group } & NSp-T & NSp-E & Sp-T & Sp-E \\
\hline $\begin{array}{l}\text { Proportion } \\
\begin{array}{l}\text { Solving } \\
\text { Deductively }\end{array}\end{array}$ & .00 & .08 & .75 & .42 \\
N & 12 & 12 & 12 & 12 \\
\hline
\end{tabular}


did act as a unit, Group NSp-E would be expected to outperform all other groups, since, for it, both goal and problem materials lead toward solution. That Group NSp-E did not outperform all other groups indicates that these two aspects of the problem situation function independently. Indeed, in the present instance, the tendency to respond to the elevated presentation may have interfered with the tendency to respond on the basis of a goal analysis, and vice versa.

\section{REFERENCES}

BERLYNE, D. E. Structure and direction in thinking. New York: Wiley, 1965.
CAMPBELL, D. T. Blind variation and selective retention in creative thought as in other knowledge processes. Psychological Review, 1960,67, 380-400. COHEN, J. An alternative to Marascuillio's "large sample multiple comparisons" for proportions. Psychological Bulletin. $1967,67,199-201$

DAVIS, G. A. Current status of research and theory in human problem solving. Psvchological Bulletin, 1966, 66, 36-54 deGROOT, A. D. Thought and choice in chess. New York: Basic Books, 1965.

DUNCKER, K. On problem solving. Psychological Monographs, 1945, 58 (Whole No. 270).

K A TON A. G. Eine kle in Auschaungsaufgabe. Psychologische Forschung, 1927, 9, 159-162.

MAIER, N. R. F. Reasoning in humans. III The mechanisms of equivalent stimuli and of reasoning. Journal of Experimental Psychology, 1945, 35, 349-360.

REID, J. W. An experimental study of analysis of the goal in problem solving Journal of General Psychology, 1951, 44. $51-69$.

\section{Semantic and associative factors in transfer}

\section{MAY F. D'AMATO and KATHY RUGGER \\ Brooklyn College, Brooklyn, N.Y. 11210}

Two experimental groups learned A-B then A-B'. For both groups, A and B were identical, and $B^{\prime}$ was equally associatively related to $B$ to a low degree. In addition, for one of the experimental groups, $B^{\prime}$ was related in meaning to $B$. Initial transfer results showed significant superiority for the semantically related over the associatively related list. Comparisons with control groups receiving C-D then $A-B^{\prime}$ revealed early positive transfer for the semantically related list and no transfer effect for the associatively related list.

When the $\mathrm{A}-\mathrm{B}, \mathrm{A} \cdot \mathrm{B}^{\prime}$ paradigm is employed, in which $B$ and $B^{\prime}$ are related in meaning, significant positive transfer has been reported (e.g., Slamecka, 1967). It has been suggested that the facilitation in such cases is not due to the semantic relatedness but rather to an underlying associative relatedness (Bastian, 1961). If associative relatedness is assumed to be the effective variable, it would be expected that equal amounts of associative relatedness would produce equal effects with additional semantic relatedness not altering the extent of transfer. The present study seeks to test this hypothesis.

\section{MATERIALS}

Four lists of nine paired associates each were used, two for first-list learning (A-B or C-D) and two for transfer-list learning (A-BS or A-BA). Stimuli were CVCs of low intralist similarity from the $40 \%-73 \%$ level of Glaze (1928). Response items were adjectives or nouns with minimal intralist relatedness. The stimuli in Lists $A-B$ and $C-D$ were equated in Glaze values, while the responses were matched for frequency of usage (Thorndike \& Lorge, 1944), part of speech, and number of syllables. The transfer lists, A-BS and A-BA, were derived by selecting two associates for each response in A-B. These associates were chosen from the norms for discrete free association of Bilodeau \& Howell (1965) such that the associative strength between a response in $A \cdot B$ and each of its two associates was essentially equal. In addition, one of the associates was also judged to have some relatedness in meaning to its corresponding word. For example, food appearing in A-BS and mouse appearing in $\mathrm{A} \cdot \mathrm{BA}$ were associates, each with $p=.06$, to cheese, which appeared in $\mathrm{A}-\mathrm{B}$. Items related in meaning to the first-list responses were used to make up the semantically related transfer list (A-BS), while items merely associatively related made up the associatively related transfer list (A-BA). Thus, the responses of both transfer lists were equally associatively related to their corresponding responses in A-B. The responses in A-BS bore, in addition, a relatedness in meaning to them. For the most part, this semantic relatedness involved synonymity, part-whole relationships, or supraordinate-subordinate relationships. In selecting responses for A-BS and A-BA, Thomdike-Lorge frequency was controlled so that about the same number of AA, A, and 35-49 per million words appeared in each list. The relation between the part of speech involved in A-B and each transfer list was controlled as much as possible. For A-BA, five of the responses were of the same part of speech as the corresponding response in $A-B$, while there were seven such items in A-BS. The actual responses were: List A-B-cheese, color, flower, foot, girl, low, memory, quick, warm; List C-D-belt, country, corner, sleep, salt, kind, magazine, loose, new; List A-BS-food, brown, plant, toe, lady, short, recall, rapid, heat; List A-BA-mouse, bright, pretty, ball, scout, bridge, fond, sharp, soft. The responses in C-D did not appear as associates to A-B responses in the norms of Bilodeau \& Howell (1965). Furthermore, a group of $57 \mathrm{Ss}$, not otherwise participating in the experiment, was presented with a list of 17 words from among which the C-D responses were selected. Four different orders of these 17 words were used in a test of continued association (Bilodeau \& Howell, 1965). Results indicated that, with one exception, the responses in A-B, $A-B S$, and A-BA did not appear among the three continued associates to each response in $C-D$.

\section{SUBJECTS}

A total of 60 volunteers from introductory psychology courses served individually as Ss. There were $15 \mathrm{Ss}$ in each of the four conditions. They were assigned randomly within blocks of four, each condition being represented once in each block.

\section{PROCEDURE}

All lists were presented with a Kodak Carousel slide projector paced by Hunter timers at a $3: 3$ rate with a 6-sec intertrial interval. Four different orders of presentation were used for all lists. Learning was to a criterion of two successive perfect trials for the first list. Transfer lists were presented for five anticipation trials or one perfect trial, whichever occurred last. Instructions presented immediately after first-list learning informed Ss that they were merely to observe the pairs on the very first exposure to the transfer list and to try to anticipate 